\title{
Comparison of multi-distance signal level difference Hjorth descriptor and its variations for lung sound classifications
}

\author{
Achmad Rizal $^{1}$, Risanuri Hidayat ${ }^{2}$, Hanung Adi Nugroho ${ }^{3}$ \\ ${ }^{1}$ School of Electrical Engineering, Telkom University, Indonesia \\ ${ }^{2,3}$ Department of Electrical Engineering \& Information Technology, Universitas Gadjah Mada, Indonesia
}

\begin{tabular}{l} 
Article Info \\
\hline Article historys: \\
Received Nov 19, 2018 \\
Revised Mar 30, 2019 \\
Accepted May 15, 2019 \\
\hline
\end{tabular}

\section{Keywords:}

Hjorth descriptor

Lung sound

Multidistance signal level

difference

Multiscale analysis

Signal complexity

\begin{abstract}
A biological signal has the multi-scale and signals complexity properties. Many studies have used the signal complexity calculation methods and multiscale analysis to analyze the biological signal, such as lung sound. Signal complexity methods used in the biological signal analysis include entropy, fractal analysis, and Hjorth descriptor. Meanwhile, the commonly used multiscale methods include wavelet analysis, coarse-grained procedure, and empirical mode decomposition (EMD). One of the multi-scale methods in the biological signal analysis is the multi-distance signal level difference (MSLD), which calculates a difference between two signal samples at a specific distance. In previous studies, MSLD was combined with Hjorth descriptor for lung sound classification. MSLD has the potential to be developed by modifying the fundamental equation of MSLD. This study presents the comparison of MSLD and its variations combined with Hjorth descriptor for lung sound classification. The results showed that MSLD and its variations had the highest accuracy of $98.99 \%$ for five lung sound data classes. The results of this study provided several alternatives for multi-scale signal complexity analysis method for biological signals.
\end{abstract}

Copyright (C) 2019 Institute of Advanced Engineering and Science. All rights reserved.

\section{Corresponding Author:}

Achmad Rizal,

School of Electrical Engineering,

Telkom University,

J1 Telekomunikasi, Trs. Buah Batu, Bandung 40287, Indonesia.

Email: achmadrizal@telkomuniversity.ac.id

\section{INTRODUCTION}

Multi-scale analysis for biological signals is essential in the biological signal analysis [1] as it is believed that the multi-scale analysis can extract information in the signal. Various multi-scale methods developed by other researchers include coarse-grained procedure [2], wavelet analysis [3], and others. A biological signal is generated from a complex biological system with various inputs and outputs where the inputs-outputs relation has not been thoroughly explained [4]. The complex system generates a complex signal, which is a signal with no proper mathematical explanation. Therefore, various complexity signal calculation methods have been developed to quantify the properties of the complex signals.

Several approaches have been developed to calculate signal complexity. Land and Damian explained three approaches to calculate signal complexity in the time domain; namely information theory, chaos theory, and Kolmogorov estimation [5]. Hjorth used statistical analysis to calculate the characteristics of biological signals [6] with the parameters of Activity, Mobility, and Complexity. Initially, Hjorth descriptor was used to analyze electroencephalogram (EEG) signals. However, the Hjorth descriptor was then used to analyze the electrocardiogram (ECG) signal [7] and lung sound [8].

In lung sound analysis, Hjorth descriptor has been calculated in various multi-scale analysis schemes to provide complexity signal multi-scale method alternatives. It was calculated in the coarse-grained procedure, in which the results were presented in [9]. Meanwhile, empirical mode decomposition (EMD) was combined 
with Hjorth descriptor in [10] to obtain higher accuracy in comparison to the combination of coarse-grained procedure and Hjorth descriptor. Hjorth descriptor was also calculated in the output of multi-distance signal level difference (MSLD) with the accuracy of up to $98.76 \%$ [11].

MSLD method is a modification of the grey level difference matrix (GLDM) method that is used for digital image analysis. As it is used for digital image analysis, the output signal of this method is an absolute value from a difference between two data samples at a specific distance. For a digital signal processing application, MSLD can be modified to generate a negative value signal. Other modification can be used to improve the accuracy of the MSLD method. In this study, MSLD was modified, and its performance in lung sound classification was analyzed. The proposed method is expected to be an alternative for analysis of lung sound or other biological signals.

\section{RELATED WORKS}

This study discusses the calculation of signal complexity in multi-scale for feature extraction in lung sound analysis. Multi-scale signal complexity analysis attracts many researchers since multi-scale entropy (MSE) method emerges [2]. MSE has been used to calculate the complexity of ECG signal in multi-scale using the coarse-grained procedure and sample entropy. The results showed that MSE could be used to distinguish normal and abnormal ECG signals. In another study, MSE has been used to recognize lung sound in alveolitis patients [12]. The results showed that MSE extracted better features than spectral features. Several other studies used the multi-scale fractal dimension and multi-level wavelet entropy packet for lung sound analysis $[13,14]$. The results showed that multi-scale processes increased classification performance compared to a single-scale method.

Some researchers used different approaches to analyze lung sounds. Sengupta et al. used cepstralbased statistical features for pulmonary sound classification, inspired by the speech processing method [15]. The study was conducted on 30 subjects with cases of normal, crackle, and wheeze lung sounds. Using ANN as a classifier, the proposed method produced better results compared to the discrete wavelet transform method that was widely used by other researchers $[16,17]$. Meanwhile, Bardou et al. focused on the use of convolutional neural networks (CNN) for lung sound classification [18]. The features used were MFCC and local binary pattern (LBP) characteristics extracted from the spectrogram. The results obtained showed that CNN's performance was better than SVM.

Hjorth descriptor was also used to calculate lung sound signal complexity [8]. Hjorth descriptor was calculated from 81 data classified into five data classes. Using K-mean clustering for data classification, the obtained accuracy was 77\%. In another study, a combination of coarse-grained procedure and Hjorth descriptor was used to calculate multi-scale signal complexity in lung sound [9]. This method was tested in the same lung sound data with the previous method, and the highest obtained accuracy was $95.06 \%$. Hjorth descriptor was also calculated in intrinsic mode function (IMF) as the result of empirical mode decomposition (EMD) process [10]. The highest obtained accuracy was at $98.8 \%$ in Activity from IMF1 to IMF10. Lastly, Hjorth descriptor was combined with a novel multi-scale method named multi-distance signal level difference (MSLD) [11]. MSLD was calculated from the absolute value of a difference between two signal samples at a specific distance $d$.

MSLD still has a potential to be developed. In this study, we proposed several MSLD variations combined with Hjorth descriptor for lung sound classification. The MSLD variations discussed in this study are MSLD-B, the multistep signal level difference (MStepLD), and multi-distance signal difference with downsampling (MSDownLD). The detailed explanation of each method is presented in the following section.

\section{MATERIAL AND METHODS}

The proposed system is displayed in Figure 1. The signal was normalized using zero mean and amplitude normalization. Then, we used the MSLD process and its variations using some distances $d$ to produced multiscale signals. Hjorth descriptor of each signal produced by the multi-distance process at distance $d$ was calculated as features of the signal. The results in the form of features of each signal were then analyzed to determine its accuracy using multilayer perceptron (MLP) to assess each multi-scale process. The following subchapters explain each process in detail. 


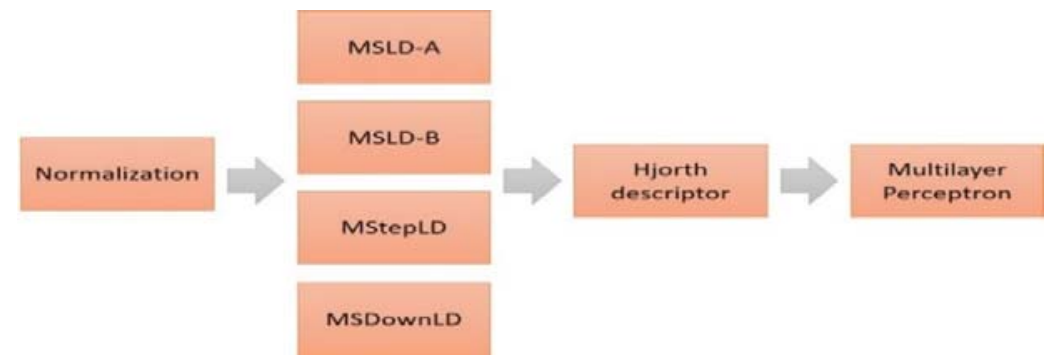

Figure 1. System design of lung sound classification using MSLD and its variations

\subsection{Lung sound data}

The input data were lung sounds with the sampling frequency of $8000 \mathrm{~Hz}$, and the length was one respiration cycle. The lung sounds were the same with data used in [8], added with the data obtained from the Internet [19], and book CD [20]. Data used in this study were:

a. Normal bronchial (22 data): this is the data of normal lung sound commonly heard in the bronchus with expiration sound tended to be longer than inspiration sound with a pause between inspiration and expiration phases [21].

b. Asthma (18 data): asthma had wheezed sound pattern. The characteristics included musical, high pitch and continuous. Here, asthma was caused by the narrow respiratory tract [22].

c. Crackle (20 data): Crackle sound had a short duration, non-musical, and discontinuous. It could be heard in booth inspiration or expiration phase. The diseases related to crackle sound include chronic bronchitis, interstitial lung fibrosis and asbestosis [23].

d. Friction pleural rub (19 data): pleural rub or friction rub had a non-musical, explosive, and commonly biphasic pattern. It is usually related to pleural inflammation or pleural tumors [22].

e. Stridor (20 data): It is one type of wheezing sound with very low frequency. It is commonly heard during inspiration and related to laryngeal stenosis or tracheal stenosis [23].

Five data classes possessed their unique characteristics. The proposed method was expected to classify input data with high accuracy.

Normalization process was conducted to overcome data variation caused by the different recording process. Firstly, zero-mean normalization was done to shift the mean value to zero. If $x(i)$ was the input signal, then zero-mean result, $\mathrm{y}(i)=x(i)-\operatorname{mean}(x)$. Secondly, amplitude normalization was done to create a signal in the amplitude range of -1 to +1 using the formula of $y(i)=x(i) / \max (|x|)$. Finally, lung sound signal was used in the multi-scale process.

\subsection{Multi-scale process}

\subsubsection{Multi-distance signal level difference}

Multi-distance signal level difference (MSLD) is a method that splits a signal into a new signal series based on a difference between two signal samples at a specific distance [10]. It can be used to observe the emergence pattern of one data sample to the other data sample at a specific distance. The MSLD formula is expressed in (1).

$$
y^{d}(i)=|x(i)-x(i+d)|, \text { for } d=1,2, \ldots, D
$$

Where $x(i)$ is the input signal, $y^{d}(i)$ is the output signal based on calculated distance $d$, and D is the maximum distance calculated to obtain $y^{d}(i)$. This study used $\mathrm{D}=20$; thus, there were 20 new signals generated from the MSLD process. At a distance of $d=1-20(\mathrm{D}=10)$, the signal variance would decrease until it tends to remain as shown in Figure 7. In previous research, Costa et al. also used D $=20$ for multiscale entropy [2]. The use of $\mathrm{D}=20$ was intended so that we could compare the results of this study with previous studies using multiscale Hjorth descriptor [9] and multiscale entropy [24].

The (1) used an absolute value, indicating that a difference between the two signals was always positive. This was conducted by considering MSLD as a modification of GLDM that was a digital image analysis method, which required all data sample to be in a positive value. However, as MSLD was used in the 1D signal analysis, then the data sample sometimes required a negative value. This study proposed another variation of MSLD as expressed in (2).

$$
y^{d}(i)=x(i)-x(i+d), \text { for } d=1,2, \ldots, D
$$


The difference between (1) and (2) is the absolute value sign. In (1), the value of $y^{d}(i)$ is always positive, but in (2), the value of $y^{d}(i)$ can be negative. To distinguish between the two MSLD methods, then (1) and (2) are called MSLD-A and MSLD-B, respectively.

\subsubsection{Multistep signal level difference}

We proposed one variation of MSLD, the multistep signal level difference or later called as MStepLD. In MStepLD, a signal in distance $d$ is calculated from the signal in distance $d-1$. The formula of MStepLD is expressed in (3).

$$
y^{d}(i)=\left|y^{d-1}(i)-y^{d-1}(i+d)\right|, \text { for } d=1,2, \ldots, D,
$$

For $d=1$, we obtained:

$$
y^{1}(i)=|x(i)-x(i+1)|
$$

where $x(i)$ is the input signal and $y^{d}(i)$ is the output signal in distance $d$.

\subsubsection{Multi-distance signal level difference with downsampling}

In one of the previous studies, MSLD was compared with the coarse-grained procedure [2]. One problem occurred was that the number of samples in MSLD was relatively constant as there was no process to reduce the number of the data sample. This is different from the coarse-grained procedure that has a reduction of data sample with the amount of $d(d=$ scale value). To imitate the property of coarse-grained procedure, then one variation of MSLD namely multi-distance signal level difference with downsampling or later called MSdownLD was proposed. The formula of MSdownLD is expressed in (5).

$$
y^{d}(i)=|x(i)-x(i+d)| \downarrow d, \text { for } d=1,2, \ldots, D
$$

The (5) is similar to (1), but with the addition of the downsampling process. The downsampling of the data samples follows the distance used. This study used downsampling from one to twenty.

\subsection{Hjorth descriptor}

Hjorth descriptor is used to calculate the complexity of a signal in the time domain [6]. It is frequently used in biological signals, such as electrocardiogram signal, electroencephalogram signal, and lung sound. There are three parameters in Hjorth descriptor: Activity, Mobility, and Complexity. These three parameters are explained as follows. For signal $x(n)$, then the first order variation $\mathrm{x}^{\prime}(\mathrm{n})$ is expressed as:

$$
x^{\prime}(n)=x(n)-x(n-1)
$$

Meanwhile, second-order variation x"(n) is expressed as:

$$
x^{\prime \prime}(n)=x^{\prime}(n)-x^{\prime}(n-1)
$$

If $\sigma_{x}$ is the standard deviation of $x(n), \sigma_{x}^{\prime}$ is the standard deviation of $x^{\prime}(n)$, and $\sigma_{x}^{\prime \prime}$ is the standard deviation of $x^{\prime \prime}(n)$, then parameters of Hjorth descriptor can be expressed as:

$$
\begin{aligned}
& \text { Activity }=\sigma_{x}^{2}=\frac{\sum_{n=0}^{N-1}(x(n)-\bar{x})^{2}}{N} \\
& \text { Mobility }=\frac{\sigma_{x}^{\prime}}{\sigma x} \\
& \text { Complexity }=\frac{\sigma_{x}^{\prime \prime} / \sigma_{x}^{\prime}}{\sigma_{x}^{\prime} / \sigma_{x}}
\end{aligned}
$$

Hjorth descriptor was calculated for all output signal of MSLD process and its variations in every scale/distance to create lung sound features.

\subsection{Classification and validation}

Indo. J. Elec. Eng. \& Inf, Vol.7, No. 2, June 2019: 345 - 356 
This study used multilayer perceptron (MLP) as classifier as it has a simple configuration with a nonlinear property. In this classifier, the weight in each input feature could make a feature with a small value that could be considered as the dominant feature. This is different from K-NN classifier where feature with large value eliminates the effect of the small-value feature. For validation, we used $\mathrm{N}$-fold cross-validation ( $\mathrm{N}$-fold $\mathrm{CV}$ ). In $\mathrm{N}$-fold $\mathrm{CV}$, data were divided into $\mathrm{N}-1$ dataset as training data and one dataset as testing data [25]. Classification process was conducted for $\mathrm{N}$ times until each dataset became a testing data. In this paper, we used $N=3$. Since we have $18-22$ data for each class data, using $N=3$ will make each data set contain $6-8$ data for each data set.

\section{RESULTS AND DISCUSSION}

One example of the wheeze signal is displayed in Figure 2. Meanwhile, the results of MSLD-A, MSLD-B, MStepLD, and MSDownLD for $d=1-5$ are shown in Figures $3-6$. The absolute value used in MSLD-A generated all output signals in a positive value, different from MSLD-B generating some signals with positive and negative values. The output signal of MStepLD was different from MSLD-A because the input signal for the next process in MStepLD is the output signal in the previous step. The output signal of MSdownLD seemed similar to MSLD-A output with the reduction of the number of data sample in the downsampling process.

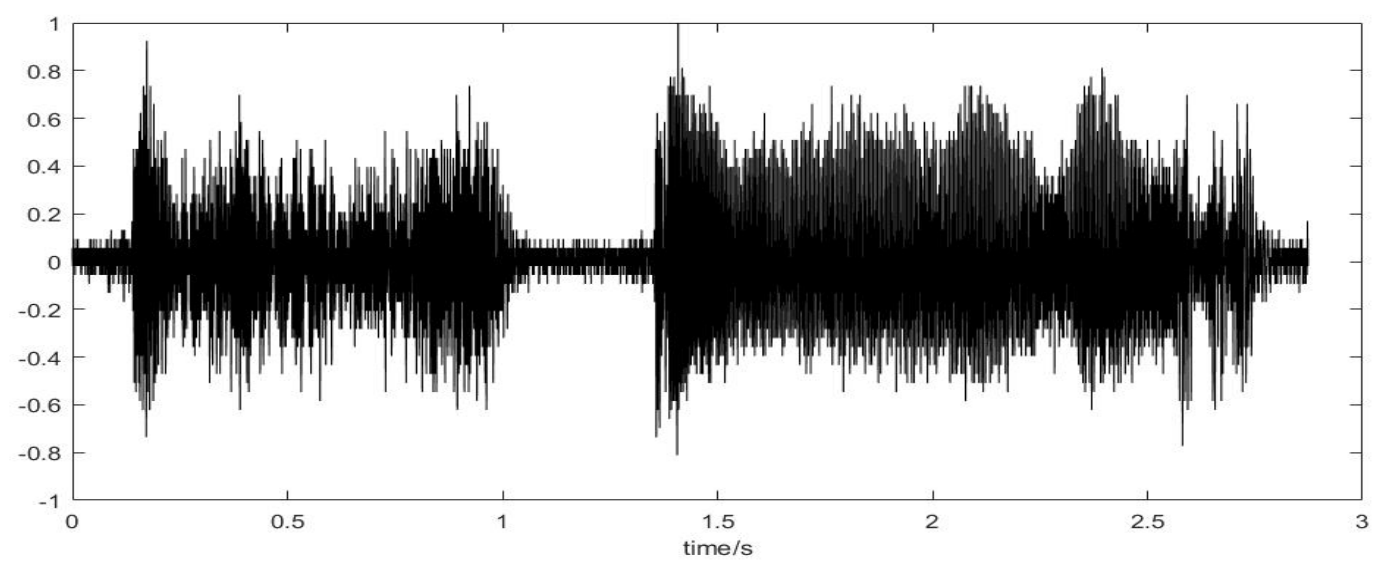

Figure 2. Example of wheeze sound

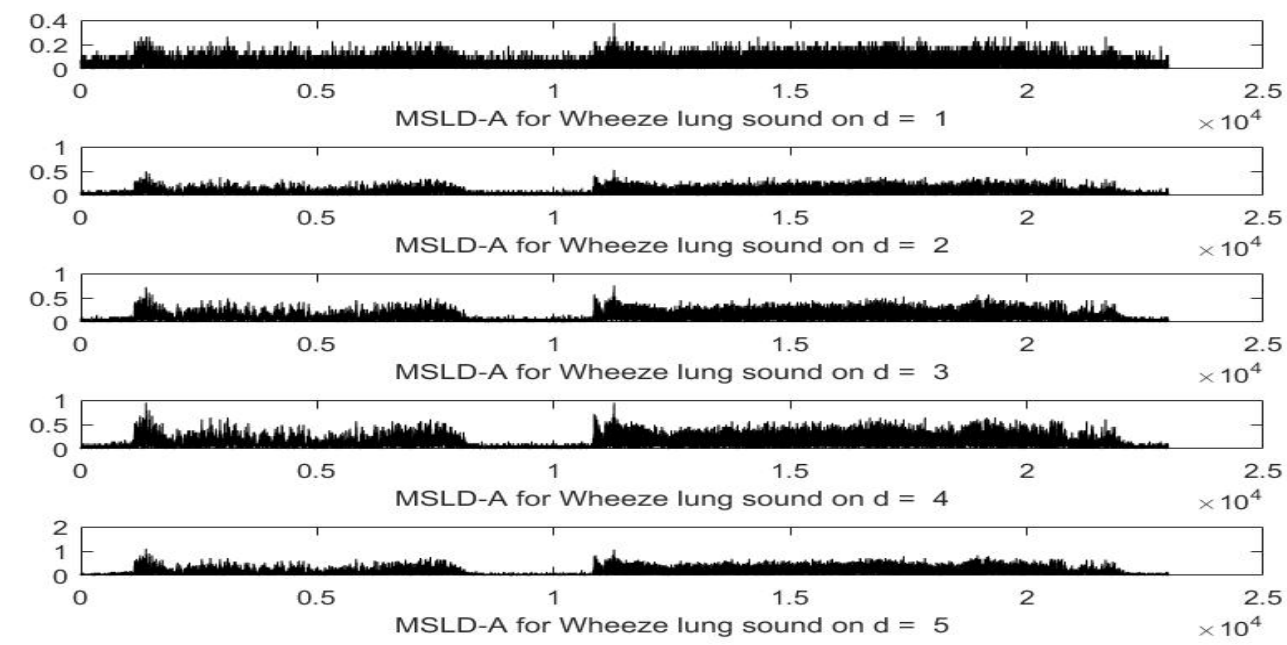

Figure 3. Result of MSLD-A process for $d=1-5$ on Wheeze sound as shown in Figure 2 


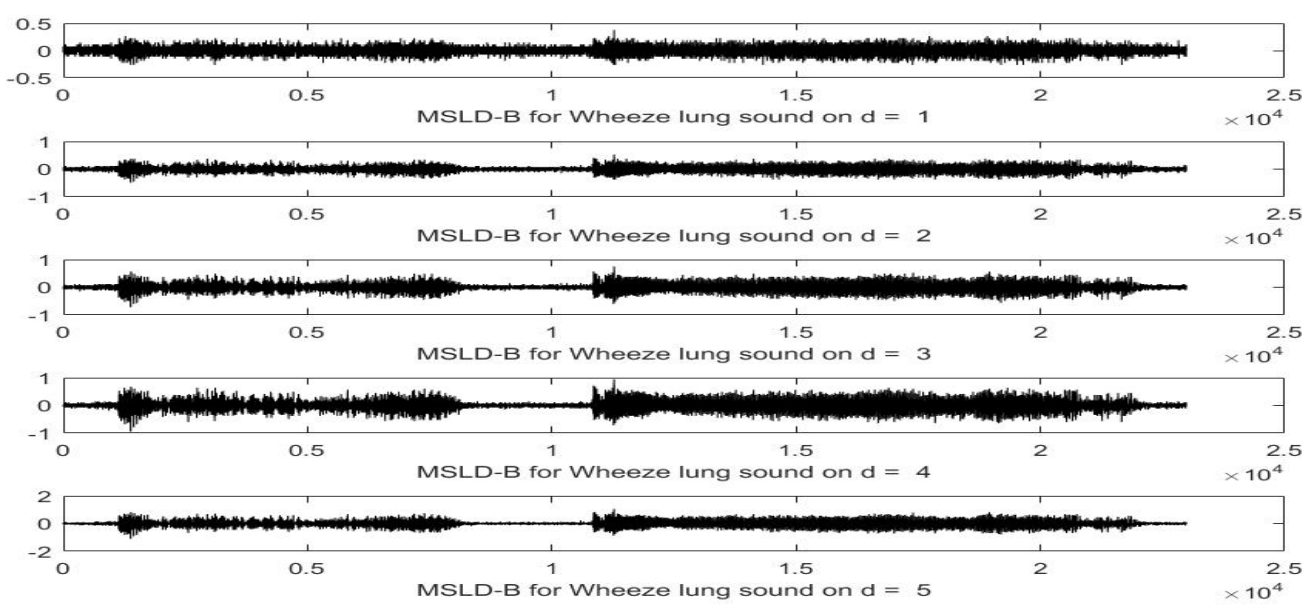

Figure 4. Result of MSLD-B process for $d=1-5$ on Wheeze sound as shown in Figure 2

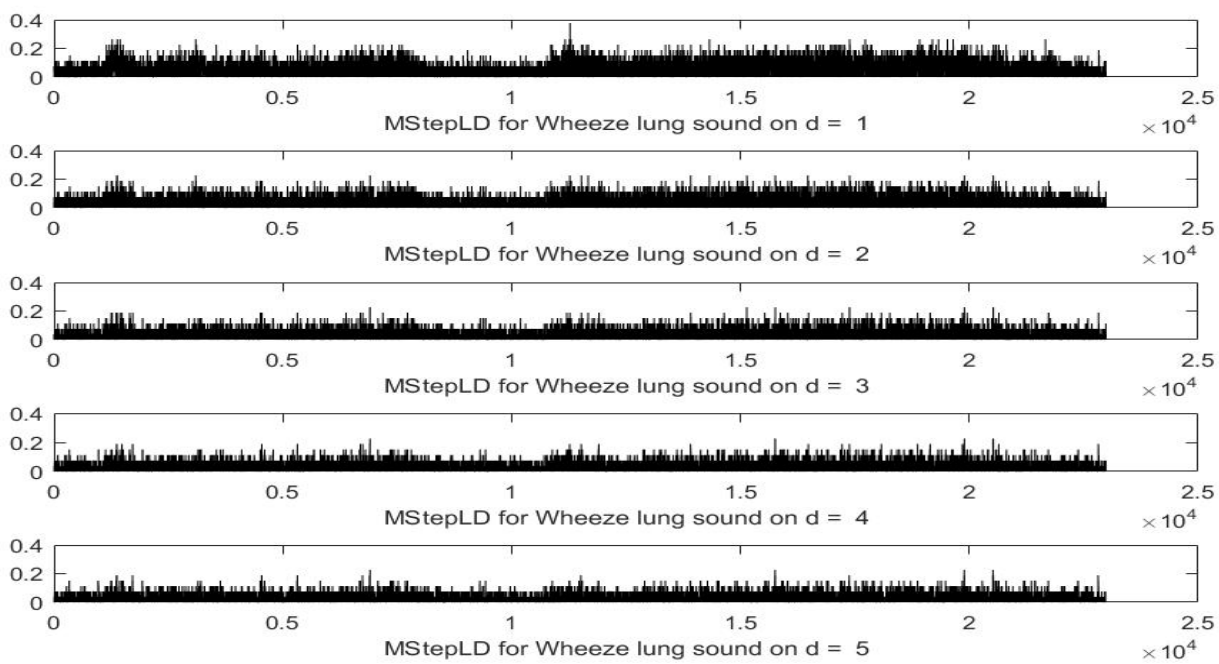

Figure 5. Result of MStepLD process for $d=1-5$ on Wheeze sound as shown in Figure 2
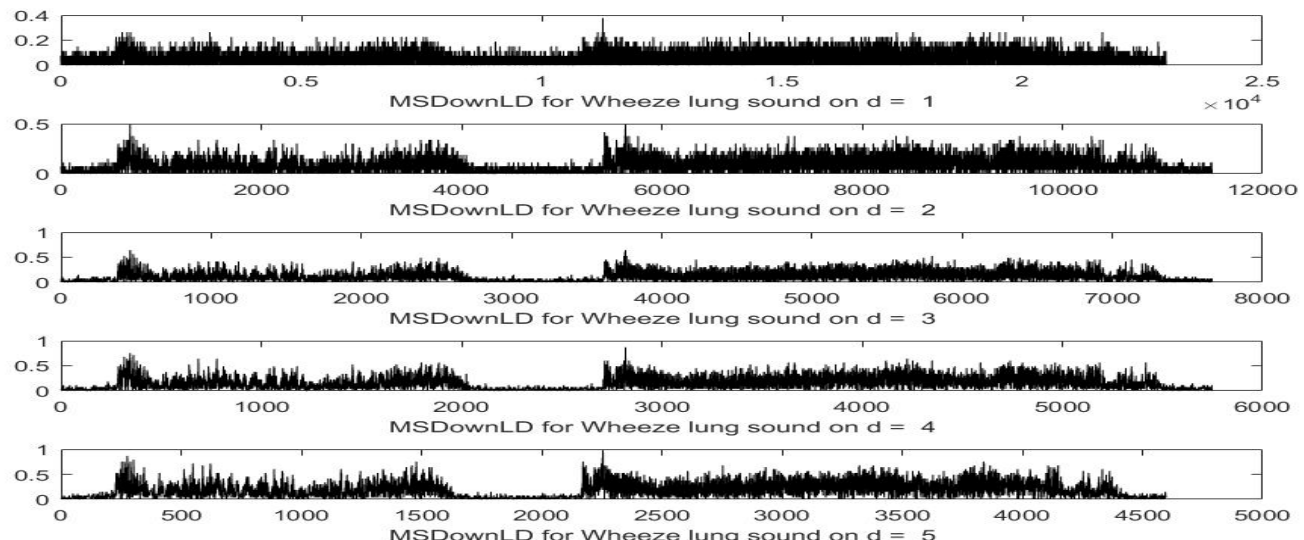

Figure 6. Result of MSDownLD process for $d=1-5$ on Wheeze sound as shown in Figure 2 


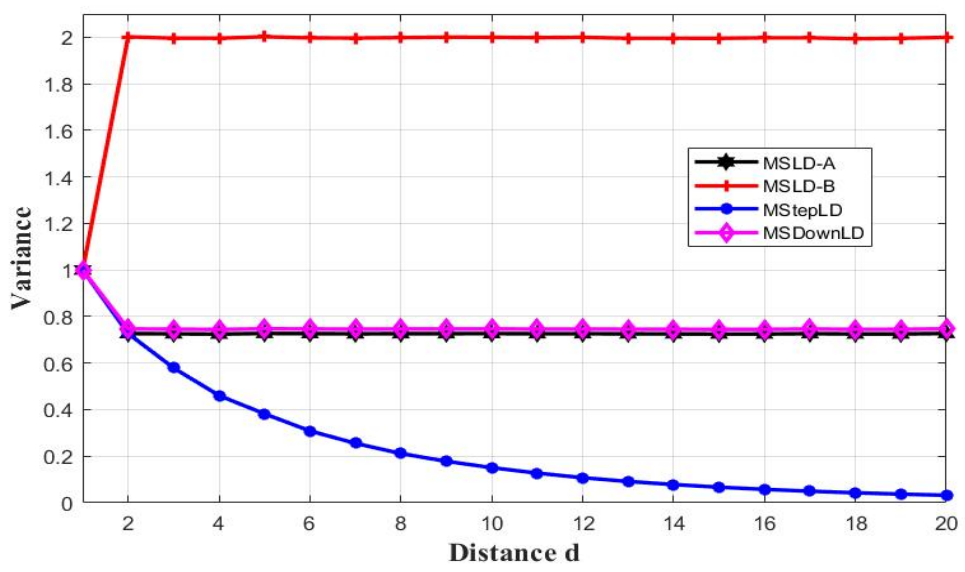

Figure 7. Comparison of variance for each process on additive white Gaussian noise

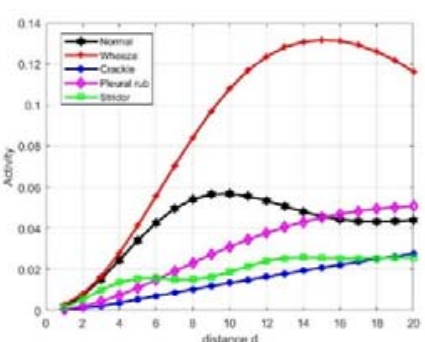

(a)

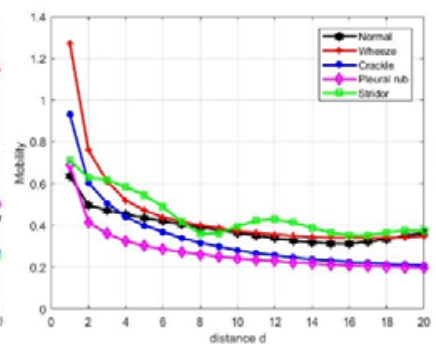

(b)

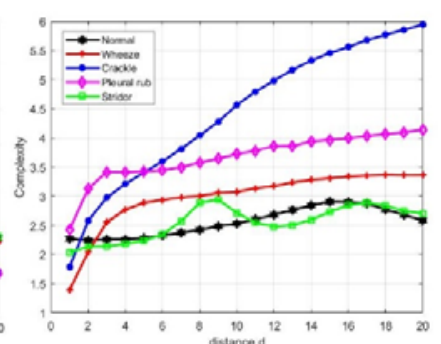

(c)

Figure 8. Hjorth descriptor on MSLD-A for typical lung sound, (a) Activity, (b) Mobility, and (c) Complexity

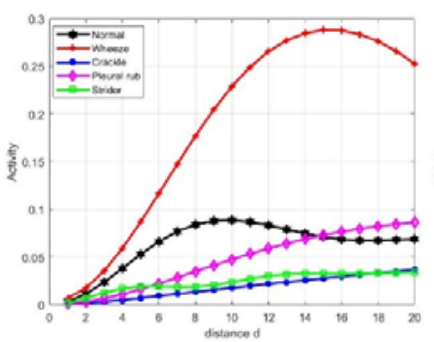

(a)

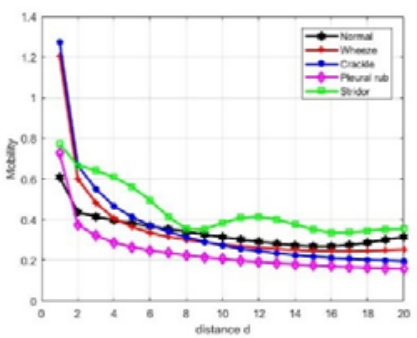

(b)

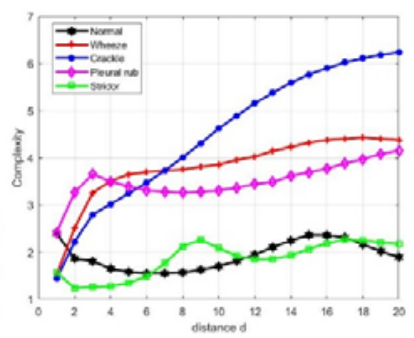

(c)

Figure 9. Hjorth descriptor on MSLD-B for typical lung sound, (a) Activity, (b) Mobility, and (c) Complexity

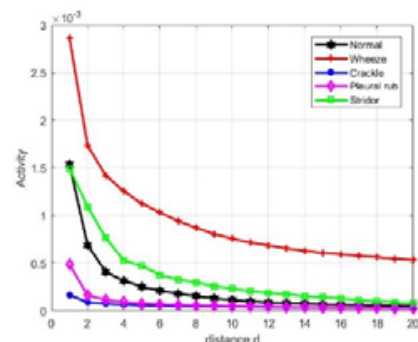

(a)

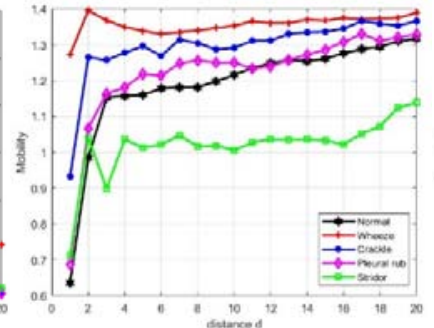

(b)

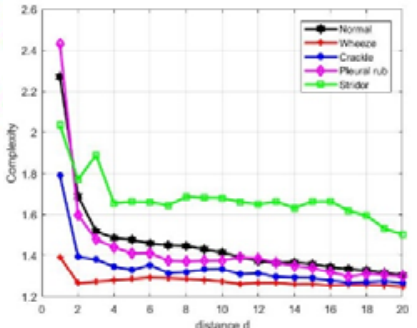

(c)

Figure 10. Hjorth descriptor on MStepLD for typical lung sound, (a) Activity, (b) Mobility, and (c) Complexity

Comparison of multi-distance signal level difference Hjorth descriptor and its variations (Achmad Rizal) 


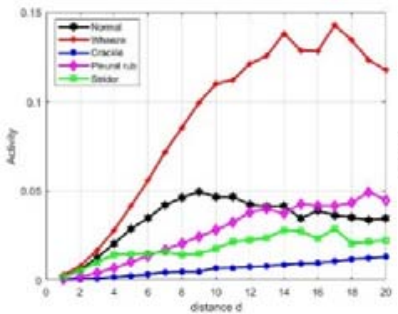

(a)

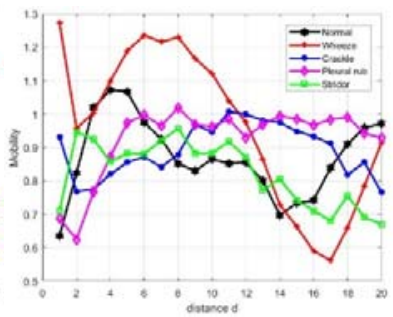

(b)

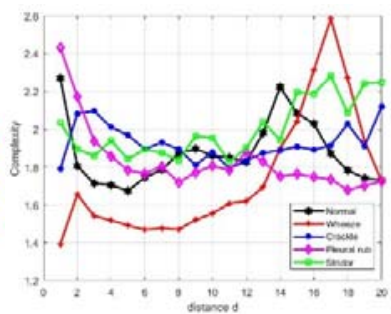

(c)

Figure 11. Hjorth descriptor on MSDownLD for typical lung sound, (a) Activity, (b) Mobility, and (c) Complexity

The difference between outputs of MSLD and its variations was observed using additive white Gaussian noise (AWGN) signal with standard deviation $=1$ and mean $=1$. The change of signal variance in each process for $d=1-20$ can be seen in Figure 7 in which MSLD-A and MSDownLD had the same variance, while MStepLD had a declined variance as the distance $d$ increased. On the contrary, MSLD-B had increased variance initially and remained constant as the distance $d$ increased. In MSLD-A and MSDownLD, the variance was declined in $d=1$ because the signal range was changed from -1 to +1 due to the absolute sign. In the next process, there was no variance change because the new signal was generated from the absolute value of the signal sample difference in distance $d$. In MSLD-B, the variance remained constant $=2$ because no absolute sign caused signal variance to increase twice. As in MSLD-A and MSDownLD, the following variance was constant. For MStepLD, the signal in $d=\mathrm{n}$ was calculated in signal $d=\mathrm{n}-1$, which made the variance decreased as $d$ increased.

The results of Hjorth descriptor calculation of each multi-scale scheme and every data class are displayed in Figures $8-11$. MSLD-A and MSLD-B had the Activity, Mobility, and Complexity graphs that were relatively similar with a different magnitude as shown in Figure 8 and Figure 9. This was caused by the fact that both processes had the same input but with a different absolute sign. The Hjorth descriptor value in MSLD-B was higher than that of MSLD-A

Figure 10 displays the Hjorth descriptor of MStepLD. The Activity value was decreased as $d$ increased since the variance value also decreased as $d$ increased. This was similar to Figure 7 . Meanwhile, Mobility and Complexity values were relatively high because there was a division process in the equation. Results in Figure 11 showed that MSDownLD had Activity value similar to MSLD-A, but the Mobility and Complexity values were different. Mobility and Complexity involved first-order, and second-order derivatives in the calculation, which made their values in MSDownLD different from MSLD-A.

The accuracies of the four methods are displayed in Figures $12-15$. Testing was conducted with 20 Hjorth descriptor values in distance $d=1-20$; then it was reduced to $d=1-15$, and so on as displayed in the figures. Initially, all Hjorth descriptor parameters were used to form 60 features, and then each Hjorth descriptor parameter was tested for its accuracy. In Fig. 12 - Fig 15, term "All" refers to three parameters of Hjorth descriptor. "All" means that in the classification, we used activity, mobility, and complexity at the same time as features for each multiscale process output. The best accuracy here was defined as the highest accuracy with the lowest number of features.

Figure 12 shows that in MSLD-A, the use of 60 features (three Hjorth descriptor parameters and distance $d=1-20$ ) yielded the highest accuracy of $96.97 \%$. However, if only the Complexity parameter that was used in the distance $d=1-15 \%$, the accuracy reached $98.99 \%$. This meant that using only one Hjorth descriptor parameter in MSLD-A was adequate to reach the accuracy up to $98.99 \%$. The accuracy tended to decrease as the number of features decreased in distance $d$.

The accuracy of MSLD-B is illustrated in Figure 13. The highest accuracy was $97.98 \%$ obtained in Complexity parameter in distance $d=1-10$. Decreasing distance $d$ lower the accuracy, except when all Hjorth descriptor parameters were used, where its accuracy increased to $96.97 \%$. In MSLD-B, the use of three Hjorth descriptor parameters in distance $d=1$ (three features) could reach an accuracy of $96.97 \%$. This value had only one error difference compared to Complexity with ten features. However, the use of three features was not selected for not meeting the predetermined best result selection criteria.

MStepLD had the highest accuracy of $97.98 \%$ as displayed in Figure 14. This result was obtained by Complexity in distance $d=1-4$. The use of a higher number of features yielded in lower accuracy. This result was similar to MSDownLD in Figure 15. The use of all Hjorth descriptor parameters in distance $d=1-5$ yielded $98.99 \%$ accuracy. Decreasing distance $d$ reduced accuracy. 
MSLD-A and MSDownLD achieved the best accuracy. MSLD-A obtained the best accuracy for the feature using Complexity with distance $d=1-15$ and MSDownLD using Activity, Mobility, and Complexity with distance $d=1-5$. Both methods used 15 features with $98.99 \%$ accuracy. These results were better than the highest accuracy of MSLD-B and MStepLD that only reached $97.98 \%$. The use of distance $d=1-20$ did not ensure higher accuracy, but reducing distance $d$ to the optimum value generated better accuracy. Determining the optimum distance $d$ to yield the best result could only be done by trial and error. In practice, Determination of optimum distance was done by observing to signal fluctuations. For cases of lung sounds, signals change rapidly with signal envelope change slowly because the respiratory rate is around 12-20 times per minute. Using a sampling frequency of $8000 \mathrm{~Hz}$, the use of distance $\mathrm{d}=1$ is equivalent to $0.125 \mathrm{~ms}$ and will only result in a relatively small amplitude change. We can see this in Figure 3 - Figure 6 . To see a significant signal shift, we can use $d>10$ or equivalent to $1.25 \mathrm{~ms}$.

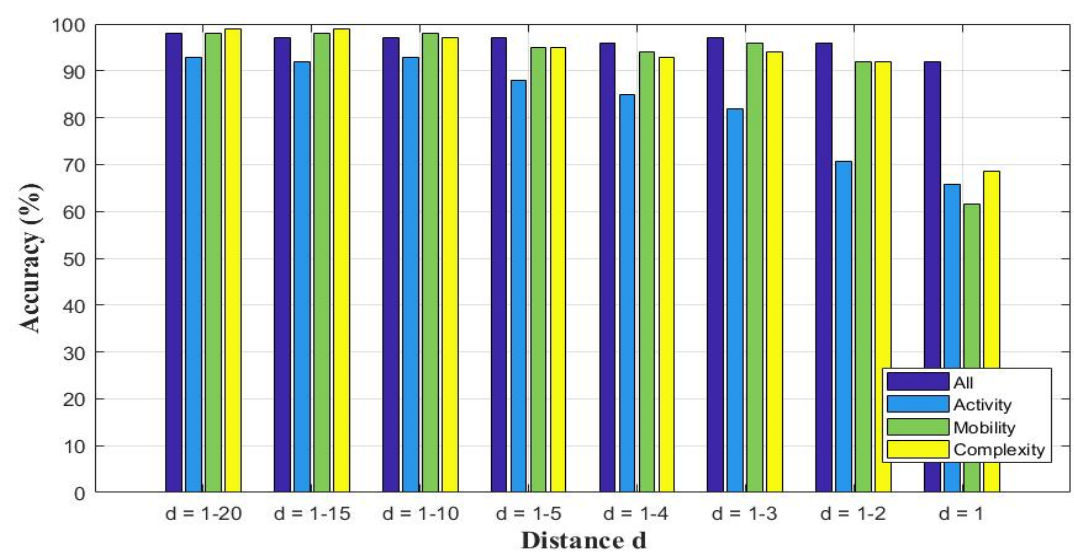

Figure 12. Accuracy of MSLD-A Hjorth descriptor for various distance $d$

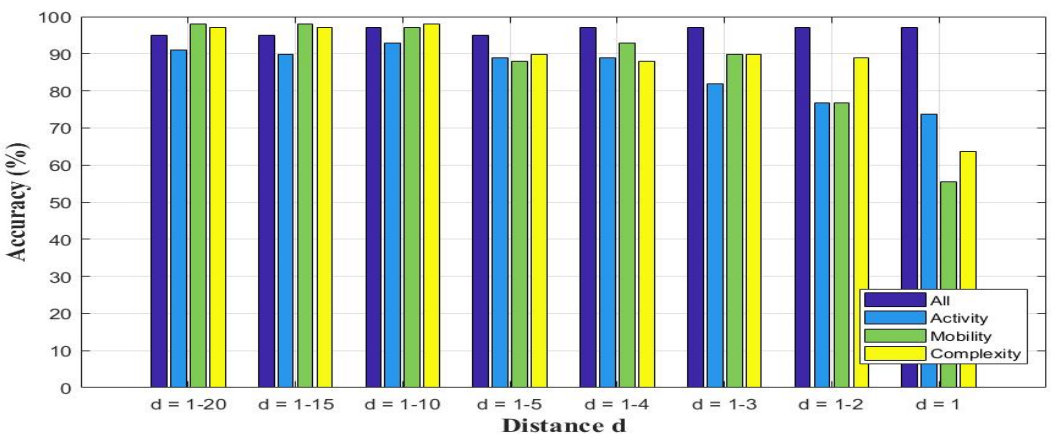

Figure 13. Accuracy of MSLD-B Hjorth descriptor for various distance $d$

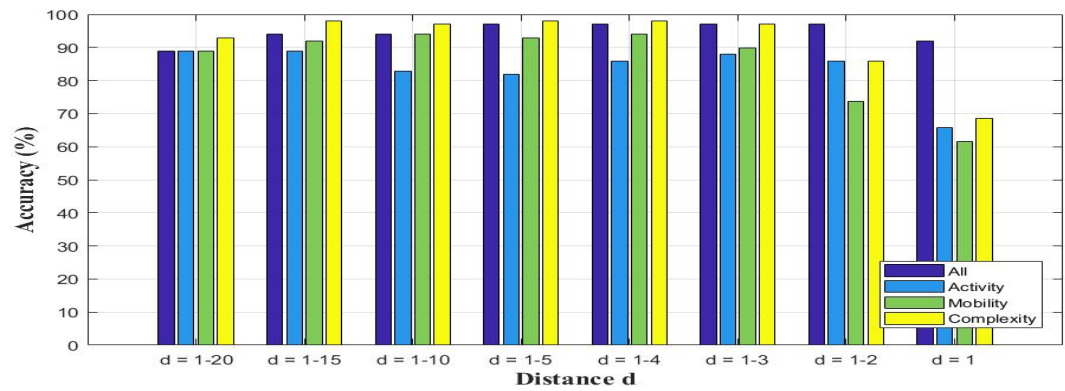

Figure 14. Accuracy of MStepLD Hjorth descriptor for various distance $d$

Comparison of multi-distance signal level difference Hjorth descriptor and its variations (Achmad Rizal) 


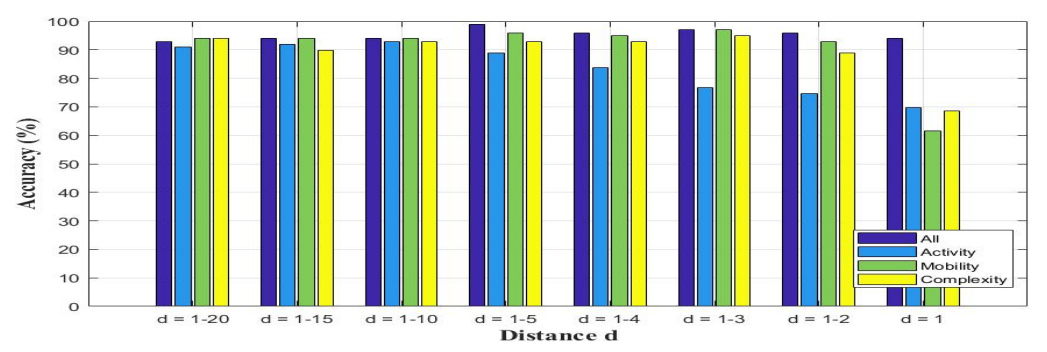

Figure 15. Accuracy of MSDownLD Hjorth descriptor for various distance $d$

Table 1. Comparison of MSLD Hjorth descriptor and its variations with previous methods

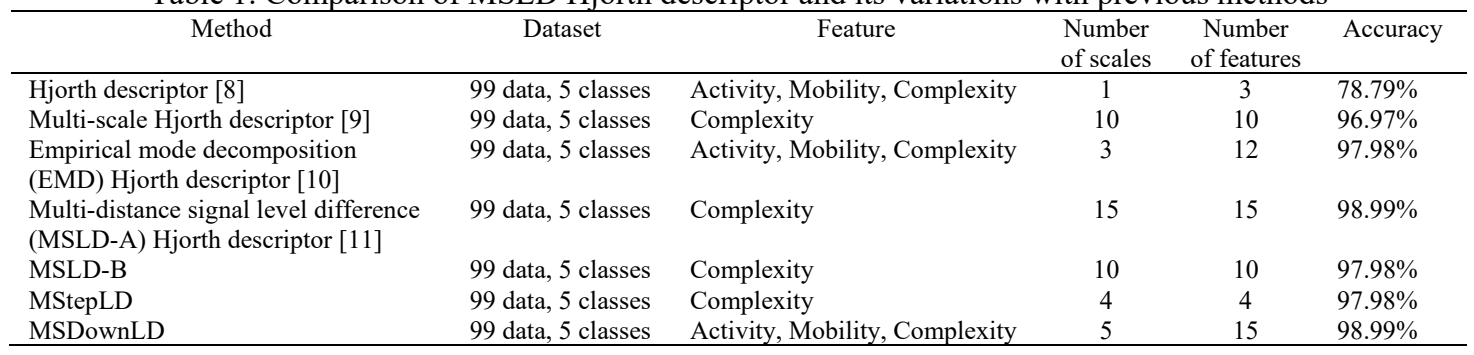

Compared with other studies regarding lung sound classification using Hjorth descriptor, MSLD-A Hjorth descriptor and MSDown Hjorth descriptor methods had better accuracy. The comparison of the results of this study and previous studies displayed in Table 1. It shows that MSLD-A and MSDownLD had the best accuracy among other methods. Both methods used 15 features with different Hjorth descriptor parameter and distance that were used. MSLD-A used Complexity parameter with distance $d=1-15$, while MSDownLD used all three Hjorth descriptor parameters in distance $d=1-5$. The difference in the computation time for both methods is not a problem because the processors used nowadays are very advanced. The next three methods with the same accuracy are EMD Hjorth descriptor, MSLD-B Hjorth descriptor, and MStepLD Hjorth descriptor. Of these three methods, MStepLD is the best selection as it has the lowest number of features to obtain the highest accuracy. In contrast, EMD Hjorth descriptor is the last choice as it has more features and the possibility of the shift of IMF value from EMD results caused by the data shift [19].

In this study, MSLD and its variations that were combined with Hjorth descriptor yielded high accuracy for lung sound classification. In previous studies, MSLD and its variations could be combined with other signal complexity calculation methods such as fractal method [26], entropy method, and others. Testing using other biological signals could also be conducted to analyze the performance of this method such as in EEG [27] or ECG [28].

\section{CONCLUSION}

This study presents several variations of the MSLD method for lung sound classification with one proposed method of MSLD-B with no absolute sign. The other variations are MStepLD that uses the results from previous steps to calculate signal in the next distance $d$, and MSDownLD that involves the downsampling process. Of four methods, it was only MStepLD yielding a decreased signal variance, while the other three methods had a constant variance. The analysis of lung sound data accuracy showed that MSDL-A and MSDownLD had the highest accuracy reaching 98.99\% using 15 features. This result is better than other methods in this study and even in the previous studies. Future works could use a larger dataset with more data classes to obtain more comprehensive results.

\section{ACKNOWLEDGMENTS}

This work has been financially supported by the Ministry of Research, Technology, and Higher Education of the Republic of Indonesia under Penelitian Disertasi Doktor Scheme no: 014/PNLT3/PPM/2018 


\section{REFERENCES}

[1] M. Costa and A. Goldberger, "Generalized multiscale entropy analysis: application to quantifying the complex volatility of human heartbeat time series," Entropy, vol. 17, pp. 1197-1203, 2015.

[2] M. Costa, A. L. Goldberger, and C. K. Peng, "Multiscale entropy analysis of biological signals," Phys. Rev. E - Stat. Nonlinear, Soft Matter Phys., vol. 71, pp. 1-18, 2005.

[3] A. Rizal, R. Hidayat, and H. A. Nugroho, "Multiresolution modified grey level difference for respiratory sound classification," Adv. Sci. Lett., vol. 23, no. 5, 2017.

[4] H. Kitaoka, R. Takaki, and B. Suki, "A three-dimensional model of the human airway tree," J. Appl. Physiol, vol. 87, pp. 2207-2217, 1999

[5] B. Land and E. Damian, "Measuring complexity," 2005. [Online]. Available: http://www.nbb.cornell.edu/neurobio/land/PROJECTS/Complexity/. [Accessed: 23-Jan-2015].

[6] B. Hjorth, "The technical significance of time domain descriptors in EEG analysis," Electroenchepalography Clin. Neurophysiol., vol. 34, pp. 321-325, 1973.

[7] A. Rizal and S. Hadiyoso, "ECG signal classification using Hjorth descriptor," in 2015 International Conference on Automation, Cognitive Science, Optics, Micro Electro-Mechanical System, and Information Technology (ICACOMIT), 2015, no. 2, pp. 87-90.

[8] A. Rizal, R. Hidayat, and H. A. Nugroho, "Determining lung sound characterization using Hjorth descriptor," in International Conference on Control, Electronics, Renewable Energy and Communication (ICCEREC), 2015, pp. 20-23.

[9] A. Rizal, R. Hidayat, and H. A. Nugroho, "Multiscale Hjorth descriptor for lung sound classification," in International Conference on Science and Technology 2015, vol. 160008, p. 160008, 2016

[10] A. Rizal, R. Hidayat, and H. A. Nugroho, "Lung sound classification using empirical mode decomposition and the Hjorth descriptor," Am. J. Appl. Sci., vol. 14, no. 1, pp. 166-173, 2017.

[11] A. Rizal, R. Hidayat, and H. A. A. Nugroho, "Hjorth descriptor measurement on multidistance signal level difference for lung sound classification," J. Telecommun. Electron. Comput. Eng., vol. 9, no. 2, pp. 23-27, 2017.

[12] S. Charleston-Villalobos, L. Albuerne-Sanchez, R. Gonzalez-Camarena, M. Mejia-Avila, G. Carrillo-Rodriguez, and T. Aljama-Corrales, "Linear and nonlinear analysis of base lung sound in extrinsic allergic alveolitis patients in comparison to healthy subjects," Methods Inf. Med., vol. 52, no. 3, pp. 266-276, Apr. 2013.

[13] A. Rizal, R. Hidayat, and H. A. Nugroho, "Fractal dimension for lung sound classification in multiscale scheme," J. Comput. Sci., vol. 14, no. 8, pp. 1081-1096, 2018.

[14] A. Rizal, R. Hidayat, and H. A. Nugroho, "Comparison of multilevel wavelet packet entropy using various entropy measurement for lung sound classification," Int. J. Adv. Comput. Sci. Appl., vol. 10, no. 2, pp. 77-82, 2019.

[15] N. Sengupta, M. Sahidullah, and G. Saha, "Lung sound classification using cepstral-based statistical features," Comput. Biol. Med., vol. 75, pp. 118-129, Aug. 2016.

[16] A. Kandaswamy, C. S. Kumar, R. P. Ramanathan, S. Jayaraman, and N. Malmurugan, "Neural classification of lung sounds using wavelet coefficients.," Comput. Biol. Med., vol. 34, no. 6, pp. 523-37, Sep. 2004.

[17] [A. Hashemi, H. Arabalibiek, and K. Agin, "Classification of wheeze sounds using wavelets and neural networks," in International Conference on Biomedical Engineering and Technology (2011), vol. 11, pp. 127-131, 2011.

[18] D. Bardou, K. Zhang, and S. M. Ahmad, "Lung sounds classification using convolutional neural networks," Artif. Intell. Med., vol. 88, pp. 58-69, 2018.

[19] "The R.A.L.E Repository." [Online]. Available: http://www.rale.ca/Repository.htm. [Accessed: 22-Jul-2015].

[20] R. L. Wilkins, J. E. Hodgkin, and B. Lopez, Lung Sounds: A Practical Guide with Audio CD, 2nd ed. Maryland Heights, Missouri: Mosby, 1996.

[21] H. Pasterkamp, S. S. Kraman, and G. R. Wodicka, "Respiratory Sounds," Am. J. Respir. Crit. Care Med., vol. 156, no. 3, pp. 974-987, Sep. 1997.

[22] A. Bohadana, G. Izbicki, and S. S. Kraman, "Fundamentals of lung auscultation.," N. Engl. J. Med., vol. 370, no. 8, pp. 744-51, Feb. 2014.

[23] S. Reichert, R. Gass, C. Brandt, and E. Andrès, "Analysis of respiratory sounds : State of the art," Clin. Med. Circ. Respirat. Pulm. Med., vol. 2008, no. 2, pp. 45-58, 2008.

[24] A. Rizal, R. Hidayat, and H. A. Nugroho, "Comparison of multiscale entropy techniques for lung sound classification," Indonesian Journal Electrical Engineering Computer and Science, vol. 12, no. 3, pp. 984-994, 2018.

[25] R. Palaniappan, Biological Signal Analysis. Ventus Publishing ApS, 2010.

[26] B. P. Harne, 'Higuchi fractal dimension analysis of EEG signal before and after OM chanting to observe overall effect on brain," Int. J. Electr. Comput. Eng. J., vol. 4, no. 4, pp. 2088-8708, 2014.

[27] M. S. A. Megat Ali, A. H. Jahidin, N. M. Tahir, and M. N. Taib, "Learning style classification via EEG sub-band spectral centroid frequency features," International Journal of Electrical Computer Engineering, vol. 4, no. 6, pp. 931-938, 2014.

[28] A. Y. Shdefat, M. Il Joo, S. H. Choi, and H. C. Kim, "Utilizing ECG waveform features as new biometric authentication method," International Journal of Electrical Computer Engineering, vol. 81, no. 2, pp. 658-665, 2018. 


\section{BIBLIOGRAPHY OF AUTHORS}
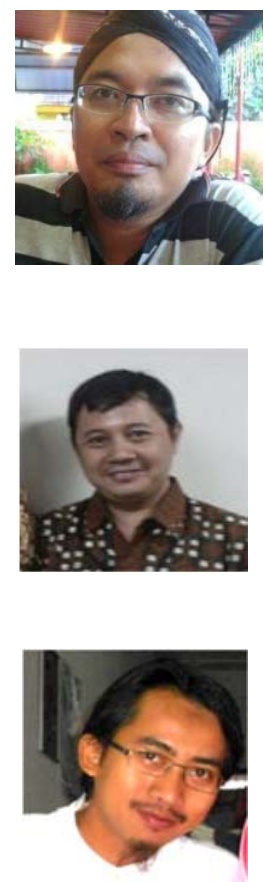

Achmad Rizal received bachelor of engineering in telecommunication engineering from STT Telkom (now, Telkom University), Bandung Indonesia in 2000. He received Master degree.in biomedical engineering from Institut Teknologi Bandung, Bandung, Indonesia in October 2006. Now, he is currently working for Ph. D degree in Universitas Gadjah Mada, Yogyakarta Indonesia on signal complexity of biomedical signal. His research interests include biomedical signal processing, biomedical image processing, biomedical instrumentation, and telemedicine

Risanuri Hidayat is an associated professor in Department of Electrical Engineering and Information Technology at Universtas Gadjah Mada, Yogyakarta, Indonesia. He received Master's degree in the field of information and communication technology achieved from Agder University College in Norway in 2002. Meanwhile, the degree of Doctor obtained from King Mongkut's Institute of technology, Ladkrabang (KMITL), Thailand in 2009 in telecommunication engineering. Dr Hidayat is the head of Digital System Lab. His research interest includes communication systems, pattern recognition and speech recognition

Hanung Adi Nugroho is an associated professor in Department of Electrical Engineering and Information Technology at Universtas Gadjah Mada, Yogyakarta, Indonesia. He received the Bachelor degree (S.T.) in electrical engineering from Universitas Gadjah Mada (UGM), Indonesia in 2001. He also received Master of Engineering degree (M.E.) in biomedical engineering from the University of Queensland (UQ), Australia in 2005. In 2012, he received his $\mathrm{PhD}$ degree in electrical and electronic engineering from Universiti Teknologi Petronas (UTP), Malaysia. He started to join as teaching staff in the Department of Electrical Engineering and Information Technology, Faculty of Engineering, Universitas Gadjah Mada (UGM) in 2002 until now. His current research interests include biomedical signal and image processing and analysis, computer vision, medical instrumentation and pattern recognition. 